\title{
NSS appelle à débat autour du projet CNRS de « zones ateliers »
}

Le programme "Environnement, vie et société " du CNRS a lancé cet été un appel d'offre centré sur la notion de "zone atelier ".

Le texte qui suit s'en inspire largement. NSS le publie pour deux raisons. Tout d'abord, parce que c'est un texte scientifique de base ; proposant une problématique d'ensemble autour de l'analyse des rapports entre écosystèmes et systèmes sociaux, il vise, en outre, à mettre en place une démarche de recherche et des dispositifs d'observation adaptés aux objectifs poursuivis, à la fois en termes d'acquisition de connaissances et de rapports à l'action. Les auteurs ont approfondi les questions soulevées dans le texte de l'appel d'offre pour en faire un texte que l'on pourrait qualifier de fondateur. Ce document appelle donc en soi le débat scientifique.

Ensuite, parce que cette action du programme "Environnement, vie et société " est appelée à durer plusieurs années (du moins peut-on l'espérer), dans la mesure où son objectif est de créer un dispositif d'observation lourd et pérenne. II s'agit donc de mobiliser des équipes scientifiques dans ce que l'on pourrait appeler le long terme. C'est un appel aux chercheurs qui est lancé. Cette initiative est d'une importance majeure pour la recherche française. C'est en effet pour elle l'occasion de se mettre en mesure de répondre à des questions qui sont d'ores et déjà posées et qui vont prendre de plus en plus d'importance dans le cadre des politiques d'environnement et de développement durable des prochaines décennies. Or elle dispose en la matière d'une solide expérience encore insuffisamment exploitée et valorisée : l'opportunité lui est ainsi offerte de remédier à cet état de fait et, par la même occasion, de se situer au niveau international en faisant valoir ses acquis.

Les questions soulevées dans ce texte et dans l'initiative scientifique dont il est en quelque sorte la charte concernent au premier chef NSS. En le publiant, la revue veut apporter son concours à la mobilisation des communautés scientifiques concernées autour de la problématique proposée et invite donc tous ceux qui sont intéressés à se faire connaître. Par ailleurs, souhaitant que cette proposition suscite le large débat qu'elle appelle, tant sur le plan proprement scientifique que du point de vue de l'organisation de la recherche, elle ouvre ses colonnes à tous ceux qui voudront y contribuer. 